\title{
Optical Variability of the Be Star o And during the Period 1975-1998: Rotational Modulation or Multimodal Pulsations?
}

\author{
G. Guerrero' ${ }^{1}$, J.P. Sareyan ${ }^{2}$, M. Alvarez ${ }^{3}$, J.Chauville ${ }^{4}$, A. Arellano \\ Ferro $^{5}$, P. Mathias ${ }^{4}$, J. Pena ${ }^{5}$, and E. Garclá ${ }^{6}$ \\ ${ }^{1}$ Osservatorio Astronomico di Brera, via E.Bianchi 46, I-22055 \\ Merate, Italy. \\ ${ }^{2}$ Observatoire de la Cote d'Azur,Departement Fresnel, B.P.229,F-06003 \\ Nice Cedex,France. \\ ${ }^{3}$ Observatorio Astronomico Nacional, UNAM,Apdo 877,Ensenada, \\ B.C.22800, Mexico. \\ ${ }^{4}$ Observatoire de Paris-Meudon,URA 335 du CNRS,F-92195 Meudon \\ Cedex, France. \\ ${ }^{5}$ Instituto de Astronomiá, UNAM, Apdo Postal 70-264, 04510, Mexico \\ D.F., Mexico. \\ ${ }^{6}$ Universidad Autónoma de Nuevo León, México
}

\begin{abstract}
Photometric observations of o And collected from 1994 to 1998 are presented. Neither multimodal pulsations nor rotational modulation seems able to explain completely the complex light curves, but a model based on the pulsations together with some type of magnetic activity could be applicable.
\end{abstract}

\section{Introduction}

So far it has not been possible to derive a physical model to explain satisfactorily the complex light variations of o And, although this object is one of the most observed Be stars.

From 1975 on we have collected a rich series of photometric data mostly at San Pedro Mártir Observatory (Baja California, Mexico).

In a paper published last year (Sareyan et al. 1998, hereafter Paper 1), some of us discussed two models, based on the pulsational and rotational hypotheses respectively, and were able to explain some aspects of the light variations of the star during the years 1975 and 1992.

Here we want to extend this comparison to the observations performed in 1994-1998.

\section{The Pulsational Model}

Using the least-squares power spectrum method of Vanicek (1971) we were able to identify a set of four frequencies showing similar values during the long period 
from 1975 to 1998 . They are reported in Table 1 with the related amplitudes, the standard deviation and the white noise level of the measures.

Note how both the frequencies and the amplitudes show significant variations from one year to the next, definitely larger than the typical errors of the frequency analysis.

The synthetic light curves, calculated on the basis of the solutions shown in Table 1, are represented in Fig.1, together with the observational data. The comparison between the r.m.s. residuals (column 6 of Table 1) and the white noise level (column 3 ) gives us an indication of the quality of the fit.

\section{The Rotational Model}

As one can see in Table 1, the first two frequencies represent approximately a double wave and give nearly always the largest contribution to the amplitude variations. Moreover, the smallest one (about $0.63 \mathrm{c} / \mathrm{d}$ ) is very similar to the rotational period of the star (see Paper 1). These facts could indicate the presence of some kind of activity (spots?) co-rotating with the photosphere. These could modulate the luminosity of the star contributing to the observed light curves.

To test this hypothesis, we also performed a least-squares fit of a double wave sinusoid. As the possible origin of such activity could be related to shortlived magnetic fields, we restricted the double wave analysis to each individual group of close observations (even inside the same observational run). Table 2 summarizes the results for the data sets from 1994 to 1998 . A similar analysis of the 1975 and 1992 data can be found in Paper 1.

\section{Preliminary Conclusions}

1. The multiperiodicity suggested in Table 1 seems able to explain some important features of the complex photometric behaviour of o And, even if the fit with the observational data cannot be considered satisfactory, in particular for the year 1996. On the other hand, the double wave analysis gives even worse results, even if we have to point out that in the first case there are 12 free parameters (frequencies, amplitudes and phases), and only 4 in the second.

2. Regarding the pulsational model (multiperiodicity), we should explain the strong variations of the frequency and amplitude values from one observational run to the next. In the framework of the rotational hypothesis, the presence of changes in the photospheric activity added to possible differential rotation with the latitude (see Paper 1) could clear up these variations.

3. A tentative model based on the presence of non-radial pulsations together with some type of magnetic activity providing a variable (stochastic?) contribution, possibly represents a viable solution. 
Table 1.

\begin{tabular}{|c|c|c|c|c|c|}
\hline Year & $\sigma$ & White noise & Frequencies & Amplitudes & RMS residual \\
\hline \multirow[t]{3}{*}{1994} & 0.023 & 0.003 & 0.643 & 0.025 & 0.003 \\
\hline & & & 0.180 & 0.016 & \\
\hline & & & 1.445 & 0.011 & \\
\hline \multirow[t]{3}{*}{1995} & 0.014 & 0.001 & 1.34 & 0.011 & 0.002 \\
\hline & & & 0.628 & 0.015 & \\
\hline & & & 1.44 & 0.017 & \\
\hline \multirow[t]{4}{*}{1996} & 0.034 & 0.001 & 1.229 & 0.034 & 0.015 \\
\hline & & & 0.622 & 0.015 & \\
\hline & & & 0.160 & 0.022 & \\
\hline & & & 1.451 & 0.020 & \\
\hline \multirow[t]{4}{*}{1997} & 0.043 & 0.010 & 1.274 & 0.028 & $0.0 \overline{8}$ \\
\hline & & & 0.652 & 0.040 & \\
\hline & & & 0.183 & 0.036 & \\
\hline & & & 1.466 & 0.020 & \\
\hline \multirow[t]{4}{*}{1998} & 0.031 & 0.015 & 1.280 & 0.010 & 0.011 \\
\hline & & & 0.632 & 0.036 & \\
\hline & & & 0.18 & 0.005 & \\
\hline & & & 1.42 & 0.003 & \\
\hline
\end{tabular}

Table 2.

\begin{tabular}{|c|c|c|c|c|c|}
\hline Year & $\sigma$ & White noise & Frequencies & Amplitudes & RMS residuals \\
\hline \multirow[t]{2}{*}{1994} & 0.023 & 0.004 & 0.601 & 0.030 & 0.007 \\
\hline & & & 1.202 & 0.012 & \\
\hline \multirow[t]{2}{*}{1995} & 0.014 & 0.001 & 0.633 & 0.010 & 0.008 \\
\hline & & & 1.266 & 0.011 & \\
\hline \multirow{2}{*}{$\begin{array}{c}1996 \\
\text { 1st part }\end{array}$} & 0.041 & 0.002 & 0.588 & 0.024 & 0.023 \\
\hline & & & 1.175 & 0.040 & \\
\hline \multirow{2}{*}{$\begin{array}{c}1996 \\
\text { 2nd part }\end{array}$} & 0.022 & 0.002 & 0.622 & 0.004 & 0.012 \\
\hline & & & 1.245 & 0.026 & \\
\hline \multirow{2}{*}{$\begin{array}{c}1996 \\
\text { 3rd part }\end{array}$} & 0.010 & 0.003 & double wave & & \\
\hline & & & not present & & \\
\hline \multirow[t]{2}{*}{1997} & 0.043 & 0.010 & 0.654 & 0.039 & 0.025 \\
\hline & & & 1.308 & 0.032 & \\
\hline \multirow[t]{2}{*}{1998} & 0.031 & 0.015 & 0.639 & 0.038 & 0.013 \\
\hline & & & 1.278 & 0.010 & \\
\hline
\end{tabular}

\section{References}

Sareyan, J. P. et al. 1998, A\&A 332, 155

Vanicek, 1971, Astrophys. Space Sci. 12, 10 